\title{
Iatrogenic Iliac Arteriovenous Fistula Management after Lumbar Discectomy Surgeries: A Case Report and Review of the Literature
}

\author{
Mihaela Salagean, Carmen Ginghina, Roxana Carmen Geana, Razvan Dragulescu, Andra Balcangiu Stroescu, \\ Ovidiu Stiru, Laura Raducu, Vlad Anton Iliescu, Adrian Tulin
}

Emergency Institute for Cardiovascular Diseases “Prof. Dr. C. C. Iliescu”, Bucharest, Romania

\section{ABSTRACT}

Iatrogenic iliac arteriovenous fistula (IAVF) is an extremely rare complication after lumbar discectomy surgery (LDS), with potentially life-threatening consequences. An IAVF results from the close anatomic relation between the iliac vessels and the last lumbar vertebrae and the corresponding discs. We report the case of a 45 -year-old woman who developed a large right IAVF 3 years after L4-L5-S1 laminectomy. The arteriovenous fistula (AVF) was successfully treated with an endovascular technique using a WALLSTENT selfexpanding stent. The postoperative period was uneventful, and the patient was discharged from the hospital in good general condition on the third postoperative day.

\section{INTRODUCTION}

Vascular injury after lumbar spine surgery is very rare, with an incidence of $\sim 1$ to 5 in 10,000 [Kubelik 2016; Papadoulas 2002]. Major vessel injury is uncommon during lumbar disk surgery (LDS); however, in $50 \%$ of patients, a small injury may go unnoticed [Hart 2003]. Often, this diagnosis is missed because of the vague nature of complaints. The time spanning from the outset of an iatrogenic iliac arteriovenous fistula (IAVF) to its diagnosis varies from months to years, and the patient is often diagnosed only after developing cardiac failure as a result of the acquired arteriovenous fistula (AVF) [Papadoulas 2002].

It is important to suspect an iatrogenic case of IAVF in a patient with lumbar spinal surgery that presents a continuous murmur in the right lower part of the abdomen. Early diagnosis and treatment can be life-saving. In patients with large IAVFs, the increase in venous return results in signs and symptoms of congestive heart failure, dyspnea, hypotension, lower-extremity swelling, and venous liver congestion. History and physical examination findings can suggest IAVF; most patients undergo computed tomography angiography (CTA) and digital subtraction angiography (DSA) examinations to confirm the potential diagnosis and rule out

Received August 27, 2020; accepted September 24, 2020.

Correspondence: Ovidiu Stiru, Emergency Institute for Cardiovascular Diseases "Prof. Dr. C. C. Iliescu," Sos. Fundeni 258, Sector 2, 022328 Bucharest, Romania; 400722207286 (e-mail: ovidiu_stiru@yahoo.com). differential diagnoses (86.4\%) [Yan 2019]. Most authors have reported that endovascular repair should be the first line of treatment of IAVF after LDS [Alshabatat 2019]. Because of the wide availability of covered stents, endovascular repair has replaced conventional surgery and is preferred in the treatment of IAVF with delayed presentation.

\section{CASE PRESENTATION}

A 45-year-old woman was referred to our clinic with palpitation complaints, exertional dyspnea, lumbar pain, right leg swelling, and diffuse abdominal pain, which was progressively

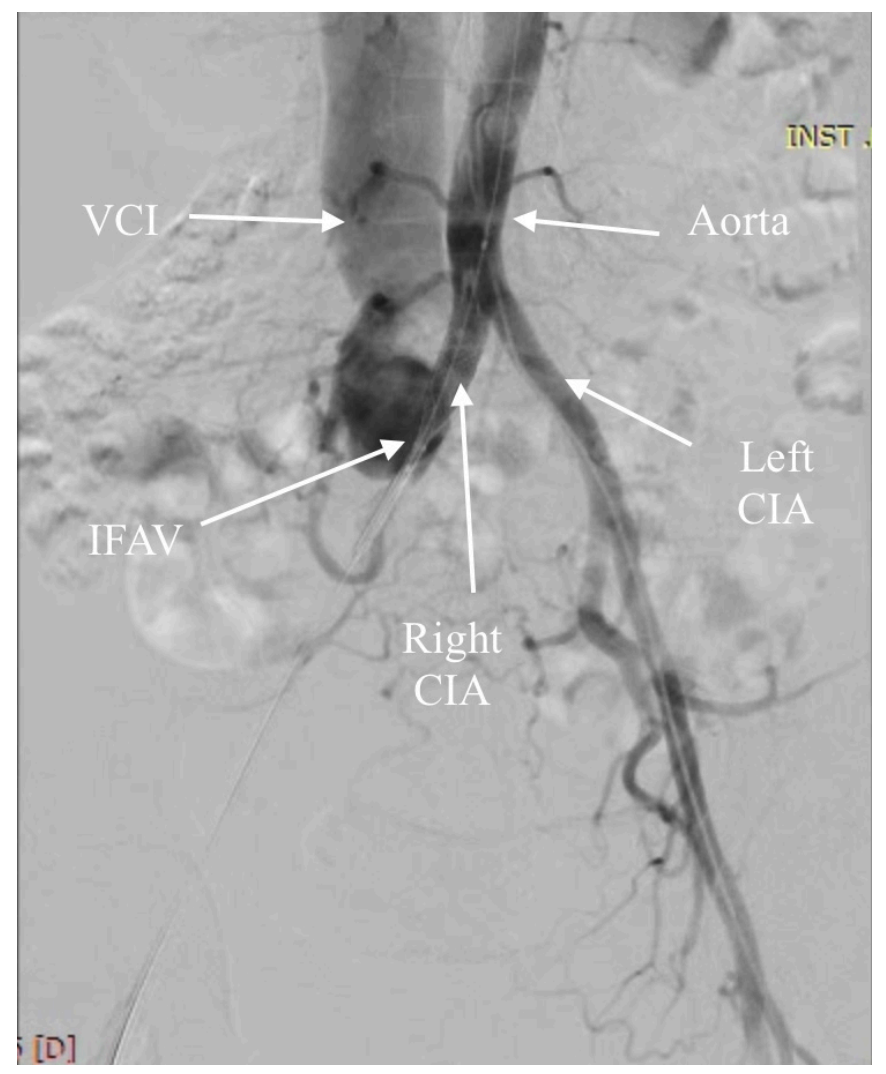

Figure 1. DSA image reveals AVF between the RCIA and RCIV, $2.5 \mathrm{~cm}$ distal to the aortic bifurcation. RCIA indicates right common iliac artery; $\mathrm{RCIV}$, right common iliac vein. 


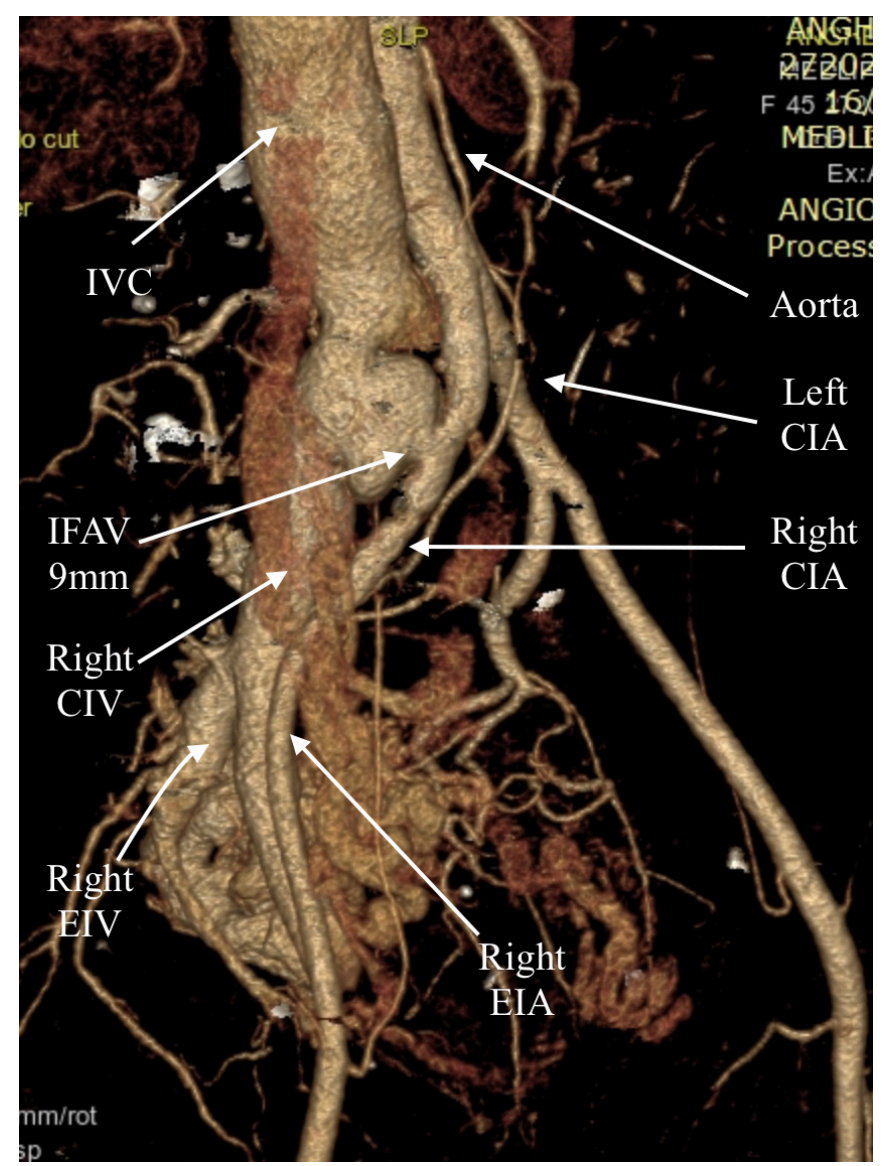

Figure 2. 3-dimensional CT angiogram showing the connection between the right common iliac artery and the right common iliac vein. AVF, 9 $\mathrm{mm}$. RCIA indicates right common iliac artery $(12 \mathrm{~mm})$; RCIV, right common iliac vein $(30 \mathrm{~mm})$.

accentuated for the past few months. She had had an L4-L5-S1 lumbar discectomy 3 years earlier. The patient had no medical history for deep venous thrombosis or ischemic heart disease. Physical examination at admission revealed congestive heart failure, findings confirmed by chest radiography, bilateral marked lower-limb edema, prominent right femoral pulse, and distended abdominal veins with massive thrill on abdominal palpation. A continuous murmur over the lower abdomen wall, in the right groin and the lumbar area, was detected at auscultation. The rest of the clinical examination findings were normal.

Based on the patient's symptoms, a history of spinal surgery, and physical findings, an IAVF was suspected. Doppler ultrasound revealed arterialized flow within the right common iliac and femoral veins. The electrocardiogram showed a normal sinus rhythm. Transthoracic echocardiography (TTE) showed normal ejection fraction of the left ventricle, an enlarged right atrium and right ventricle with severe tricuspid insufficiency, and increased right ventricular systolic pressure. Coronary angiography was normal. Hematologic and biochemical tests were normal except for mild thrombocytopenia, $130,000 / \mathrm{mm}^{3}$. For

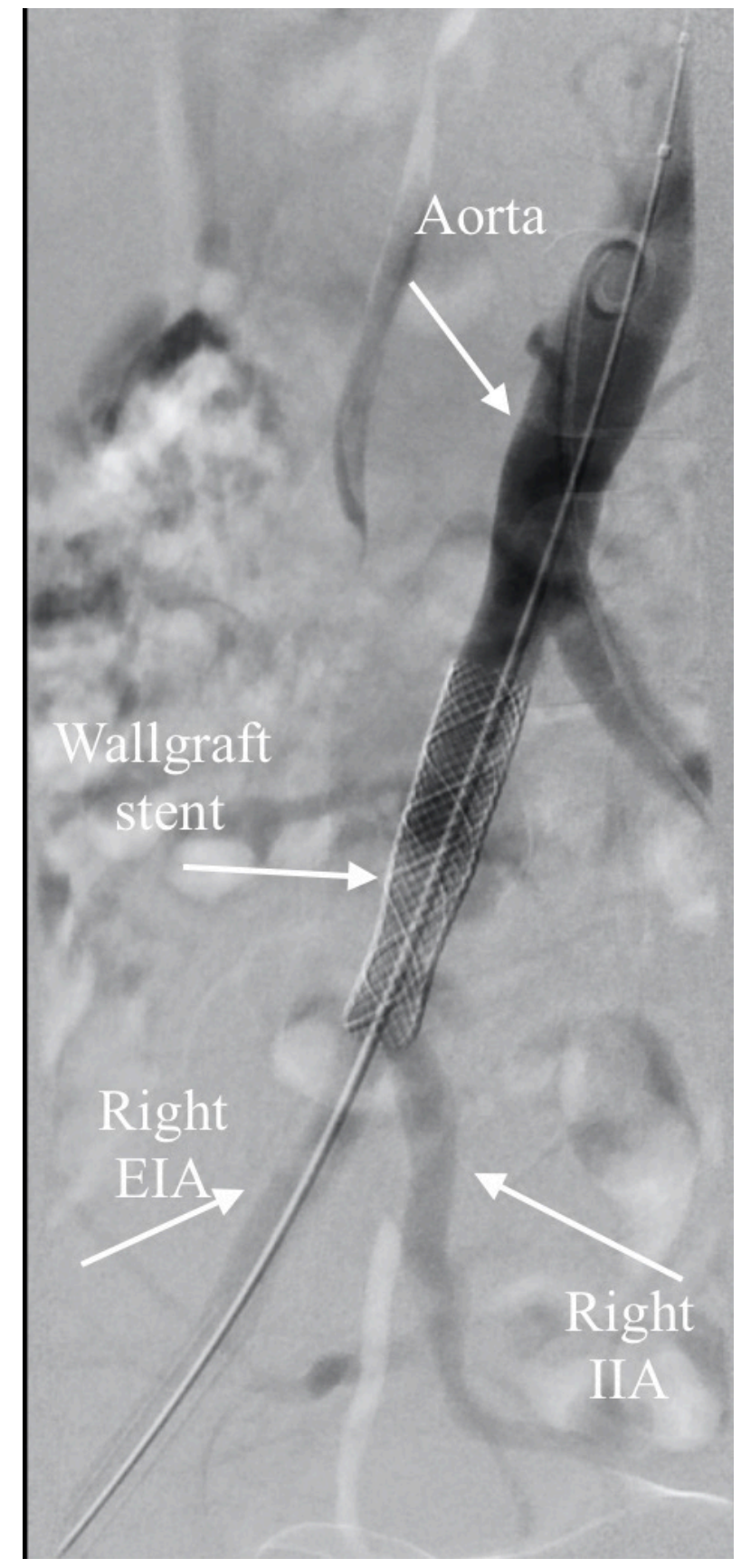

Figure 3. Post-stent pelvic DSA showing patent stent-graft across the right common iliac artery with minimum evidence of filling of the fistula.

diagnosis, we performed abdominal aorta DSA obtained via left femoral approach, which showed communication between the right common iliac artery and right common iliac vein, distal to the aortic bifurcation (Figure 1). CTA (Figure 2) with 3-dimensional reconstruction examination confirmed high-flow communication between the right common iliac artery and right common iliac vein, at the level of L5-S1, $2.5 \mathrm{~cm}$ distal to the aortic bifurcation; a fistula with a diameter of $9 \mathrm{~mm}$; significant dilation of the 


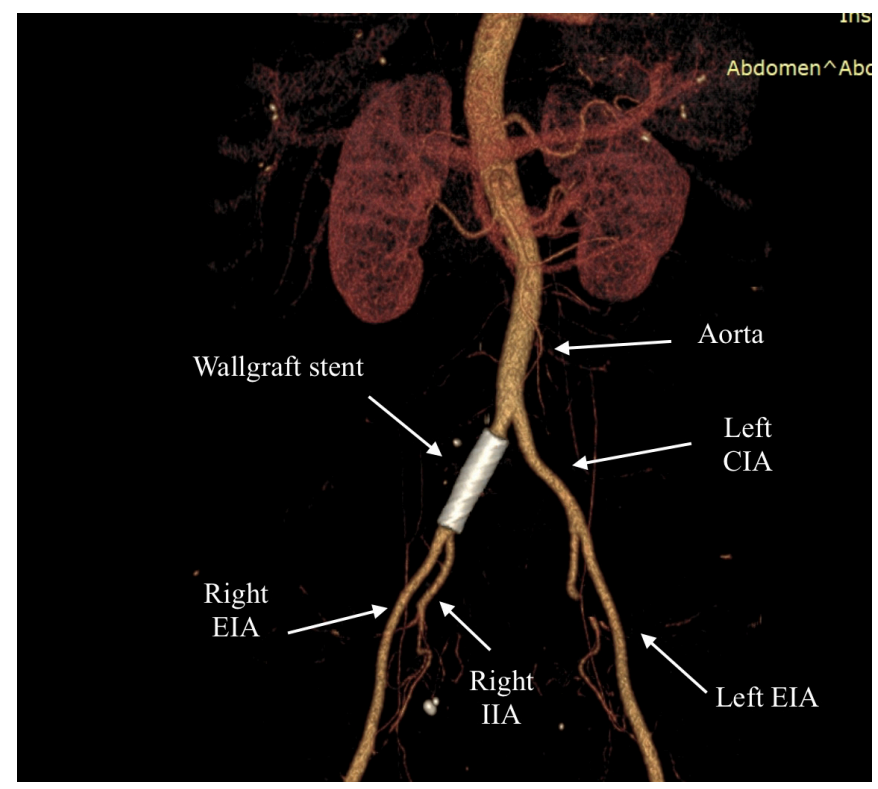

Figure 4. Post-stent/graft deployment pelvic DSA showing the stent in position and the permeability of RIIA. RIIA, right internal iliac artery, REIA, right external iliac artery.

common right iliac vein and cava vein; and a multitude of dilated and tortuous pelvic veins.

The endovascular repair offered complete resolution and was our treatment method. The vascular team's evaluation recommended stent deployment in the common right iliac artery with complete coverage of the AVF to achieve an adequate sealing zone without coverage of the internal iliac artery. Doppler ultrasound of peripheral arteries in our case found a suitable femoral artery for endograft procedures, and there were no contraindications. After general anesthesia, with invasive monitoring of blood pressure (right radial artery), through a percutaneous right common femoral artery approach, a self-expanding covered WALLGRAFT endoprosthesis (Boston Scientific MediTech, Natick, MA) was placed in the right iliac arterial system through an 11-F sheath and deployed across the fistula. Our method of inserting the stent/graft has been previously described in detail [Stiru 2018, Stiru 2019].

Subsequent intraoperative angiography showed minimal venous right iliac opacification (Figure 3). No postprocedural complications occurred. On the first day after the procedure, the abdominal murmur was no longer audible. The patient was discharged 3 days after the procedure and was advised to return periodically for follow-up. At 1 week, Doppler ultrasound examination showed that the iliac AVF persisted, but with low flow. At 1 month, Doppler ultrasound and CT angiogram confirmed the anatomic correction, with no evidence of a persistent arteriovenous fistula (Figure 4). The patient's symptoms of heart failure were resolved after 4 months. A follow-up echocardiogram confirmed resolution of the dilated heart chambers and moderate tricuspid insufficiency. At 3 years postoperatively, the patient was found to be doing well without further complications.

\section{DISCUSSION}

Iatrogenic vascular injury complications after LDS include laceration, arteriovenous fistula, and pseudoaneurysm. Patients suffering from iatrogenic vascular injury complications might not have any symptoms, or the symptoms may occur after a long time. Papadoulas et al [2002], after reviewing publications during 1965 to 2001, found 66 cases reported for IAVF of the iliac artery after an LDS. An important role in the occurrence of IAVF after LDS is played by a wide vascular bed separated by the anterior longitudinal ligament from the lumbar spine. As a result, vascular complications occur when the anterior longitudinal ligament and the adjacent arteries and veins are perforated by dissecting instruments of the disc space. $73 \%$ of IAVFs occur at the L4-L5 disk space, and the remainder occur at the L5-S1 space [Geraghty 2009]. Jarstfer et al [1976] found that almost $29 \%$ of AVFs involve the right iliac artery to iliac vein, and $25 \%$ of them are diagnosed $\geq 1$ year after the LDS.

If an IAVF is formed immediately after lumbar surgery, with a small laceration including both arterial and venous tears, the patient does not develop hypotension, because arterial blood does not extravasate outside the artery, but in the adjacent vein. Gradually, in time, the flow in the fistula can increase proportional to the diameter of the fistula [Ramacciotti 2007] and, as in the case of our patient, cardiac failure begins to develop and progressively increases in severity. Often, an IAVF is suspected after development of lower-extremity edema and cardiac failure associated with symptoms of tachycardia, dyspnea on exertion, orthopnea, cardiomegaly, and in some severe cases, ascites with hepatomegaly and claudication or leg ischemia by steel syndrome in the leg supplied by the injured artery [Kwinta 2020].

IAVF is difficult to treat surgically. Treatment of IAVF carries high mortality and morbidity rates, between $5 \%$ and $10 \%$ (up to $34 \%$ ), because of dilated vein collaterals and deep location of iliac vessels [Osman 2017; Pawar 2018]. Classic surgical repair techniques in the IAVF are either suturing the communication from inside after arteriotomy or venotomy or AVF division followed by lateral vein and artery suturing. In the case presented here, surgery repair was considered extremely risky because of the frailty of AVF and iliac vein, with the risk of substantial blood loss perioperatively. Surgical bypass grafting aorto-/right iliac with the exclusion of IAVF and the iliac system was another option [Hart 2003], but its benefits may be more anecdotal than factual. In 1996, McCarter et al first reported the endovascular treatment of an IAVF after lumbar disc surgery [McCarter 1996], and the endograft approach provided the solution, with low morbidity [Noland 2017]. The challenge of this case was choosing an appropriate stent graft length that did not cover the origin of the internal iliac artery, to avoid the need to embolize it before endostent deployment. In addition to the technical success achieved with endovascular treatment of the IAVF, systemic anticoagulation to prevent venous thrombosis and pulmonary thromboembolism was administered. Close congestive heart failure, dyspnea, hypotension, lower-extremity swelling, dilated vein collaterals, and venous liver congestion were rapidly and completely reversed. 


\section{CONCLUSION}

Endovascular treatment with self-expanding stents has proved to be a safe method for correction of acquired IAVF, is the only definitive treatment for secondary congestive heart failure, and can be considered the first choice in iatrogenic lumbar discectomy arterial injury.

\section{REFERENCES}

Alshabatat A, Srayrah S, Aljfoot S, et al. Endovascular treatment of iatrogenic iliocaval fistula post lumbar disc surgery. J Surg Case Rep 2019;2019:rjz313.

Geraghty S, Durham JD, Levy JM, et al. Endovascular repair of an arteriovenous fistula after intervertebral disk surgery: Case report. J Vasc Interv Radiol 2009;20:1235-1239.

Hart JP, Wallis F, Kenny B, et al. Endovascular exclusion of iliac artery to iliac vein fistula after lumbar disk surgery. J Vasc Surg 2003;37:1091-1093.

Jarstfer BS, Rich NM. The challenge of arteriovenous fistula formation following disk surgery: A collective review. J Trauma 1976;16:726-733.

Kubelik D, Morellato J, Jetty P, et al. Endovascular repair of a chronic AVF presenting as post-partum high output heart failure. EJVES Short Rep 2016;31:19-22.

Kwinta BM, Myszka AM, Bigaj MM, et al. Iatrogenic common iliac vessel injury during routine degenerative lumbar spine surgery: Report of 2 cases and review of literature. World Neurosurg 2020;137:111-118.
McCarter DH, Johnstone RD, McInnes GC, et al. Iliac arteriovenous fistula following lumbar disc surgery treated by percutaneous endoluminal stent grafting. Br J Surg 1996;83:796-797.

Noland S, Espinoza CA, Dvorak JD, et al. Endovascular repair of iatrogenic iliocaval fistula causing high-output cardiac failure after spine fusion. Ann Vasc Surg 2017;45:262.e1-e5.

Osman Ocal, Bora Peynircioglu, Gonca Eldem, et al. Iliac arteriovenous fistulas after lumbar spinal surgery. Turk J Emerg Med 2017;17:109-111.

Papadoulas S, Konstantinou D, Kourea HP, et al. Vascular injury complicating lumbar disc surgery. A systematic review. Eur J Vasc Endovasc Surg 2002;24:189-195.

Pawar P, Sharma A, Raju R, et al. Iatrogenic arteriovenous fistula after lumbar disc surgery: Case reports and review of literature. Indian J Vasc Endovasc Surg 2018;5:63-65.

Ramacciotti E, Galego SJ, Gomes M, et al. Fistula size and hemodynamics: An experimental model in canine femoral arteriovenous fistulas. J Vasc Access 2007;8:33-43.

Stiru O, Geana CR, Pavel et al. Descending thoracic aortic aneurysm rupture treated with thoracic endovascular aortic repair in a patient with peripheral artery disease. Heart Surg Forum 2018;21:E112-E116.

Stiru O, Valeanu L, Parasca CA, et al. Case report: Aberrant left vertebral artery management in traumatic transection of the aortic isthmus. Heart Surg Forum 2019;22:E481-E485.

Yan GW, Li HW, Yang GQ, et al. Iatrogenic arteriovenous fistula of the iliac artery after lumbar discectomy surgery: A systematic review of the last 18 years. Quant Imaging Med Surg 2019;9:1163-1175. 